\title{
Tomografia eletromagnética poço-a-poço usando os regularizadores de Suavidade Global e de Variação Total
}

\author{
Julielson.M.Santana, Victor.C.T.de Souza, UFPA, Brazil
}

Copyright 2014, SBGf - Sociedade Brasileira de Geofísica.

Este texto foi preparado para a apresentação no VI Simpósio Brasileiro de Geofísica, Porto Alegre, 14 a 16 de outubro de 2014. Seu conteúdo foi revisado pelo Comitê Técnico do VI SimBGf, mas não necessariamente representa a opinião da SBGf ou de seus associados. É proibida a reprodução total ou parcial deste material para propósitos comerciais sem prévia autorização da SBGf.

\section{Resumo}

Neste trabalho, descrevemos o problema direto e inverso de tomografia eletromagnética poço-a-poço. A geometria do modelo possui simetria azimutal, o que simplifica significativamente o processo de modelagem do problema direto e inversão, reduzindo um equação originalmente tensorial 3-D para uma forma escalar bidimensional. No problema direto usamos - método de elementos finitos para a solução numérica da equação de Helmholtz. Já no problema inverso discutimos o uso de três funcionais estabilizadores: Suavidade Global (GS), Variação Total (TV) e Igualdade Absoluta (Al). O primeiro funcional usa uma suavização na norma $L_{2}$, enquanto o segundo usa uma suavização na norma $L_{1}$, que aceita variacões abruptas entre os parâmetros adjacentes. Nossos resultados mostram que o uso do método TV obteve uma boa estimativa da geometria e condutividade dos corpos, tanto para pequenos, quanto para grandes contrastes de condutividades entre os alvos e o meio encaixante. Observamos também que o regularizador de Variação Total apresentou uma melhor estimativa dos parâmetros, comparado com Suavidade Global. Em boa parte dos modelos sintéticos utilizados neste trabalho, obtivemos uma melhor estimativa dos corpos, quando usamos regularizadores de Suavidade Global e de Variação Total com o auxílio do vínculo de Igualdade Absoluta nas bordas da malha de inversão.

\section{Introdução}

Grande maioria das investigacões geofísicos são de difícil acesso diretamente. Para obter um modelo representativo dessas regiões inacessíveis se utiliza uma representação visual ou imageamento das propriedades física destas regiões, esta representação visual é denominada tomografia. Para se obter essas representações, a partir dos dados tomográficos, utilizase técnicas denominadas de inversão, que é simplesmente um conjunto de métodos matemáticos usados para se extrair inferência úteis sobre um conjunto de medidas observacionais de algum fenômeno físico.

O conceito de Tomografia teve seu início com os estudos de radiografia para realizar diagnósticos na medicina via emissão de raio-x French et al. (1973), Schneider and French (1976). Tendo grande êxito na medicina a tomografia foi utilizada em outras áreas tecnológicas como a geofísica.

\section{Problema direto}

Um importante aspecto das ciências físicas é fazer deduções sobre parâmetros físicos dos dados. O problema direto na tomografia eletromagnética é definido pelo conjunto de medidas de campos eletromagnéticos avaliados nos receptores. Esses campos são produzidos por fontes arranjadas em uma determinada configuração e pelos campos secundários produzidos por corpos anômalos espalhadores. No problema inverso, o objetivo é reconstruir os modelos do problema direto a partir de um conjunto de medidas que formam os dados. Seguindo o trabalho de Alumbaugh and Morrison (1995). O modelo geométrico consiste de uma estrutura, cuja condutividade possui uma simetria cilíndrica, dentro de uma região homogênea com condutividade primária $\sigma^{p}$. A geometria empregada aqui, simplifica significantemente o processo de inversão, reduzindo uma equação tensorial 3-D para uma forma escalar 2-D. Desse modo pode-se conseguir uma seção transversal do modelo 3-D. Considerando a geometria

Figura 1: Exemplo da geometria 3-D utilizada na tomografia EM.

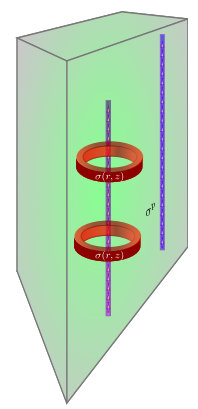

Fonte: Souza (2001)

apresentada na Figura 1. A formulação é geral para qualquer problema que explore a simetria axial e o modo transverso elétrico TE. Considerando a simetria axial do modelo, em que não existe variações na direção azimutal $\frac{\partial}{\partial \theta}=0$.

As equações de Maxwell são escritas como:

$$
\begin{gathered}
H_{z}^{p}=-\frac{1}{\mathfrak{z} r} \frac{\partial\left(r E_{\theta}^{p}\right)}{\partial r}, \\
H_{z}^{s}=-\frac{1}{\mathfrak{z} r} \frac{\partial\left(r E_{\theta}^{s}\right)}{\partial r}, \\
H_{r}^{s}=\frac{1}{\mathfrak{z}^{r}} \frac{\partial\left(r E_{\theta}^{s}\right)}{\partial z}, \\
\frac{\partial H_{r}^{s}}{\partial z}-\frac{\partial H_{z}^{s}}{\partial r}=\mathfrak{y} E_{\theta}^{s}+\Delta \mathfrak{y} E_{\theta}^{p},
\end{gathered}
$$

em que $\mathfrak{y}=\sigma, \mathfrak{z}=i \omega \mu, \Delta \mathfrak{y}=\mathfrak{y}-\mathfrak{y}^{p}=\sigma-\sigma^{p}$, onde $\sigma$ representa a condutividade tanto do meio considerado primário $\sigma^{p}$, que pode ser um espaço ilimitado, ou um semi-espaço ou em um meio estratificado, ou também pode representar condutividades anômalas a este meio primário, $\omega=2 \pi f$ é a frequência angular e $f$ é a frequência em Hertz ( $\mathrm{Hz})$. Substituindo (3) e (2) em (4) temos a equação diferencial para o modo TE axial:

$$
\frac{\partial^{2} E_{\theta}^{s}}{\partial z^{2}}+\frac{\partial}{\partial r}\left[\frac{1}{r} \frac{\partial\left(r E_{\theta}^{s}\right)}{\partial r}\right]-i \omega \mu \sigma E_{\theta}^{s}-i \omega \mu\left(\sigma-\sigma^{p}\right) E_{\theta}^{p}=0
$$

A componente primária do campo $E_{\theta}^{p}$ é gerada por um dipolo magnético num meio homogêneo, e o campo elétrico em um espaço ilimitado pode ser expressado analiticamente Ward and Hohmann (1988) como:

$$
E_{\theta}^{p}=-\frac{i \omega \mu m_{z}}{4 \pi} \frac{r}{R^{3}}\left(1+i k_{p} R\right) e^{-i k_{p} R},
$$


onde $m_{z}$ é o momento de dipolo magnético $k_{p}=\sqrt{-i \omega \mu \sigma^{p}}$ e $R=\sqrt{r^{2}+z^{2}}$. A componente primária do campo $H_{z}$ possuí solução analítica, substituindo (6) em (1) e fazendo manipulações matemáticas, obtêm-se:

$$
H_{z}^{p}=\frac{m_{z}}{4 \pi R^{5}}\left[\left(3+3 i k_{p} R-k_{p}^{2} R^{2}\right) z^{2}-\left(1+i k_{p} R-k_{p}^{2} R^{2}\right)\right] R^{2} e^{-i k_{p} R}
$$

O campo secundário $E_{\theta}^{s}$ feito pela heterogeneidade no meio encaixante é dado por:

$$
E_{\theta}^{s}=E_{\theta}-E_{\theta}^{p},
$$

A componente secundária $E_{\theta}^{s}$ é aproximada por um polinômio do tipo:

$$
E_{\theta}^{s}=E_{\theta i}^{s} \Psi_{i}+E_{\theta j}^{s} \Psi_{j}+E_{\theta k}^{s} \Psi_{k}
$$

nos nós $i, j$ e $k$ de um elemento triangular genérico de um domínio

Para calcular os campos elétricos secundários produzidos pelas heterogeneidades, a formulação pelo métodos dos elementos finitos pode ser construída aplicando um princípio variacional ou o método de Galerkin na equação diferencial (5) onde a solução desta equação é mostrada com mais detalhes em Souza (2001).

O campo desconhecido sobre cada nó da malha de discretização é aproximado por um polinômio linear em relação as coordenadas $r$ e $z$, do tipo $\varphi_{m}=\frac{1}{2 A}\left(a_{m}+b_{m} r+c_{m} z\right)$ onde o índice $m$ representa o nó no elemento triangular.

Aplicando o método de Galerkin na equação (5), obtém-se a equação matricial dos elementos finitos na forma:

$$
\mathbf{K} \varphi=\mathbf{v},
$$

onde $\mathbf{K}$ é uma matriz de rigidez global, bandeada, esparsa e simétrica, representando a geometria do modelo e as propriedades elétricas, $\varphi$ é um vetor dos valores nodais dos campos secundários desconhecidos e $\mathbf{v}$ é um vetor fonte obtido da última parte da equação (5).

\section{Problema inverso}

Problemas inversos são em regra problemas matematicamente mal-postos. Um problema é mal-posto no sentido de Hadamard quando sua solução não satisfaz a pelo menos uma das condições: existência, unicidade e estabilidade. Problemas de dados tomográficos é um problema mal posto, significa que ele não possui solução única ou estável, ou seja,existe inúmeras soluções diferentes que conseguem reproduzir as observações com uma mesmo nível de aproximação. Para resolver a questão da não unicidade e a instabilidade, somamos ao funcional que representa os dados observados um outro funcional estabilizante para introduzir informação a priori. Para minimizar o funcional não-linear resultante da soma dos dois primeiros, empregamos o método de Marquardt, que procura uma solução aproximada iterativamente. Representamos o vetor $y^{0}$ contendo o campo magnético observado no receptor como uma função da posição $z$ e dos parâmetros $\mathbf{P}$ que formam o chamado modelo interpretativo, onde consideramos como sendo aquela no qual gera os dados observados. Se tivermos $N$ observações:

$$
y_{i}^{0}=f\left(\mathbf{P}, z_{i}\right), \quad i=1,2 \ldots N
$$

onde $z_{i}$ donata as posições dos receptores. Neste trabalho, as observações será a parte real e imaginária da componente vertical do campo magnético. Os parâmetros de cada modelo são definidos pela condutividade dos elementos da malha localizados na região de imageamento. O problema de minimização do funcional estabilizante $\phi^{v}$, que contém a informação a priori e honra a informação geofísica $\phi^{o}$, pode ser resolvido através do método dos multiplicadores de Lagrange, que permite transformar esse problema no de minimização do funcional não vinculado $\tau$, denominado funcional suavizante(função objetivo), dado por:

$$
\tau(\mathbf{P})=\phi^{o}(\mathbf{P})+\mu \phi^{v}(\mathbf{P}),
$$

$\phi^{o}(\mathbf{P})$ representa o ajuste entre os dados observados e os calculados. O problema de inversão geofísica pode então ser formulado como a minimização do funcional $\phi^{v}(\mathbf{P})$, presumido de antemão ser não linear, contínuo e com derivadas contínuas de primeiras e segundas ordens em relação à $\mathbf{P}$. Assim, a formulação matemática do problema inverso usando apenas os dados geofísicos é:

$$
\min \phi^{o}(\mathbf{P})=\frac{1}{N}\left\|y^{o}-f(\mathbf{P})\right\|=\delta,
$$

sendo $\|$.$\| a norma Euclidiana e \delta$ o erro médio quadrático das realizações da variável aleatória que contamina as observações. O problema de encontrar $\hat{\mathbf{P}}$, uma estimativa de $p$ que minimize o funcional $\phi^{o}(\mathbf{P})$, é mal-posto, pois sua solução, na presença de ruído, não é única e nem estável e $\phi^{v}(\mathbf{P})$ é o funcional usado para introduzir informações a priori que ajudam a estabilizar o processo de inversão. $\mu>0$ é o parâmetro de regularização, cujo papel é controlar a influência da informação introduzida a priori. Valores pequenos de $\mu$ não permitem a estabilização da solução, enquanto valores muito grandes tornam a solução tendenciosa e impedem um ajuste aceitável.

\section{Regularização de Tikhonov}

A regularização introduzida por Tikhonov permitiu que se obtivessem para problemas mal-postos, lineares ou não lineares, soluções estáveis através do uso de informação a priori quantitativa ou qualitativa sobre a solução procurada. $\mathrm{O}$ problema bem-posto de inversão geofísica é formulado como a minimização de um funcional estabilizante $\phi^{v}$, sujeito ao ajuste, entre os dados observados e os dados calculados através do modelo interpretativo, estar dentro de uma precisão ditada pelo nível de ruído:

$$
\left\{\begin{array}{ccc}
\min \phi^{v}(\mathbf{P}) & = & \left\|W^{\frac{1}{2}} \mathbf{P}\right\|^{n} \\
& = & \mathbf{P}^{T} W p, \\
\text { sujeito a } \phi^{o}(\mathbf{P}) & = & \frac{1}{N}\left\|y^{0}-f(\mathbf{P})\right\|^{2}=\delta,
\end{array}\right.
$$

em que $W$ é uma matriz definida positiva que contém a informação a priori, chamada matriz de pesos, e o sobrescrito T denota transposição e $n$ denomina a ordem da norma euclidiana.

\section{Guass-Newton usando estratégia de Marquardt}

A solução do problema não linear é encontrada de forma interativa pelo método Gauss-Newton usando a estratégia de Marquardt (1963), para usar o método de Gauss-Newton é necessário fazer uma expansão de $\tau$ em série de Taylor no entorno da aproximação $\mathbf{P}=\mathbf{P}_{k}$ até os termos de segunda ordem, a ser chamada de $\hat{\tau}$ : Tomando-se 0 gradiente de $\hat{\tau}$ em relação a $\mathbf{P}$ e igualando-se o resultado ao vetor nulo, é possível obter uma estimativa iterativa do vetor de parâmetros necessário para atingir o mínimo de $\hat{\tau}$, ou seja,

$$
\begin{aligned}
\Delta \hat{\mathbf{P}}_{k}=-\left[\left.\nabla_{\mathbf{P}} \nabla_{\mathbf{P}}^{T}\left\{\phi^{o}(\mathbf{P})\right\}\right|_{p=p_{k}}+\left.\mu \nabla_{\mathbf{P}} \nabla_{\mathbf{P}}^{T}\left\{\phi^{v}(\mathbf{P})\right\}\right|_{\mathbf{P}=\mathbf{P}_{k}}\right]^{-1} . \\
{\left[\left.\nabla_{\mathbf{P}}\left\{\boldsymbol{\phi}^{o}(\mathbf{P})\right\}\right|_{\mathbf{P}=\mathbf{P}_{k}}+\left.\mu \nabla_{\mathbf{P}}\left\{\boldsymbol{\phi}^{v}(\mathbf{P})\right\}\right|_{\mathbf{P}=\mathbf{P}_{k}}\right], }
\end{aligned}
$$

onde podemos escrever a estimativa da seguinte forma:

$$
\hat{\mathbf{P}}_{k+1}=\hat{\mathbf{P}}_{k}+\Delta \hat{\mathbf{P}}_{k}
$$

O passo é adicionado ao valor $\mathbf{P}_{k}$ e o resultado $\mathbf{P}_{k+1}$ é substituído no lugar de $\mathbf{P}_{k}$ na iteração seguinte. Comumente, esse processo é repetido até que os valores obtidos para $\mathbf{P}_{k+1}$ sejam aproximadamente iguais aos valores obtidos na iteração anterior para $\mathbf{P}_{k}$, ou seja, até ser atingido ou se estar muito próximo do mínimo de $\tau$.

Escreveremos o gradiente $\nabla_{\mathbf{P}} \tau$ e o Hessiana $\nabla_{\mathbf{P}}\left(\nabla_{p}^{T} \tau\right)$ como as somas dos gradientes $g$ e Hessianas $H$ dos funcionais das observações $\phi^{o}$ e dos vínculos $\Phi^{v}$ :

$$
\begin{array}{ll}
g_{k}^{o}=\nabla_{\mathbf{P}} \phi^{o} \mid \mathbf{P}=\mathbf{P}_{k}, & H_{k}^{o}=\nabla_{\mathbf{P}}\left[\nabla_{\mathbf{P}}^{T} \phi^{o}\right] \mid \mathbf{P}=\mathbf{P}_{k}, \\
g_{k}^{v}=\nabla_{\mathbf{P}} \Phi^{v} \mid \mathbf{P}=\mathbf{P}_{k}, & H_{k}^{v}=\nabla_{\mathbf{P}}\left[\nabla_{\mathbf{P}}^{T} \Phi^{v}\right] \mid \mathbf{P}=\mathbf{P}_{k} .
\end{array}
$$


O valor estimado do funcional $\tau$ da seguinte maneira :

$$
\hat{\tau}(\mathbf{P})=\left.\tau(\mathbf{P})\right|_{\mathbf{P}=\mathbf{P}_{k}}+\Delta \mathbf{P}^{T}\left(g_{k}^{o}+\mu g_{k}^{v}\right)+\frac{1}{2}\left(H_{k}^{o}+\mu H_{k}^{v}\right) \Delta \mathbf{P},
$$

A matriz de derivadas segundas (Hessiana) do funcional estabilizante $\Phi^{v}$ é conhecida analiticamente ( $2 W$ se $W$ for independente de $\mathbf{P}$ ). O mesmo não ocorre com a Hessiana do funcional geofísico, sendo necessária sua avaliação numérica, - que envolve o cálculo de segundas derivadas, o que é extremamente dispendioso computacionalmente. Por isso, usase a aproximação de Gauss-Newton $\left.\nabla_{\mathbf{P}}\left[\nabla_{\mathbf{P}}^{T} \phi^{o}\right]\right|_{\mathbf{P}=\mathbf{P}_{k}} \approx 2 J_{k} J_{k}$, que vêm da substituição de $f(\mathbf{P})$ pela sua expansão de primeira ordem $f\left(\mathbf{P}_{k}\right)+J_{k}\left(\mathbf{P}-\mathbf{P}_{k}\right)$, onde $\left(J_{k}\right)^{T}=\nabla_{\mathbf{P}}[f(\mathbf{P})]_{\mathbf{P}=\mathbf{P}_{k}}^{T} \operatorname{Bard}$ (1974). A matriz $J_{k}$ é matriz de sensibilidade (Jacobiana), que consiste das derivadas parciais do funcional ajustante $f$ em relação aos parâmetros, avaliada em um conjunto particular de valores $\mathbf{P}_{k}$ para os parâmetros. . A aproximação do vetor de parâmeros na $k$-ésima+ 1 iteração passa a ser escrita da seguinte maneira:

$$
\hat{\mathbf{P}}_{k+1}=\hat{\mathbf{P}}_{k}-\left[J_{k}^{T} J_{k}+\mu W+\lambda I\right]^{-1}\left[J_{k}^{T}\left(y^{o}-f\left(\hat{\mathbf{P}}_{k}\right)\right)+\mu W \mathbf{P}_{k}\right] .
$$

O termo $\lambda$, conhecido como parâmetro de Marquardt, é ajustado automaticamente durante o processo iterativo estabelecido por Marquardt. Repeti-se esse processo até satisfazer o critério de convergência definido em termos da função $\hat{\tau}$ de um parâmetro de controle $(\varepsilon)$, que dever muito pequeno. Desse modo, foram incorporados dois critérios de parada:

- Diferença relativa da função objetivo:

$$
\text { conv }=\left|\frac{\hat{\tau}_{k}-\hat{\tau}_{k+1}}{\hat{\tau}_{k}}\right|<\text { tol }
$$

- Desajuste dos dados:

$$
\operatorname{conv}<\phi^{o} \leq t o l,
$$

sendo tol a tolerância pré-estabelecida para o desajuste dos dados. O critério 18 evita a parada do processamento caso seja satisfeito o critério 17 e o ajuste dos dados ainda possa ser melhorado, ou seja, evita que a incorporação da informação a priori, sobre a qual sempre existem incertezas, prepondere sobre a informação geofísica que é representada pelas medidas de campo. Para este trabalho o valor de tol vale $10^{-6}$.

\section{Regularizador de Igualdade Absoluta}

Para o vínculo de Igualdade Absoluta faremos a seguinte formulação:

$$
\left\{\begin{array}{c}
\min \phi^{A I}(\mathbf{P})=\|A \mathbf{P}-v\|^{2}, \\
\text { sujeito a } \phi^{o}(\mathbf{P})=\frac{1}{N}\left\|y^{0}-f(\mathbf{P})\right\|^{2}=\delta,
\end{array}\right.
$$

onde $v$ são os valores aos quias vincularemos os parâmetros e A é uma Matriz cujas linhas são formadas por zeros exceto na posição dos parâmetros vinculados conforme o exemplo abaixo em que os parâmetros $\mathbf{P}_{1}, \mathbf{P}_{2}, \mathbf{P}_{3}, \mathbf{P}_{200}$ são iguais a $0.01 \mathrm{~S} / \mathrm{m}$ :

$$
A=\left[\begin{array}{cccc}
1 & 0 & 0 \ldots & 0 \\
0 & 1 & 0 \ldots & 0 \\
. & . & . & . \\
. & . & . & . \\
. & . & . & . \\
. & . & . & . \\
0 & 0 & 1 \ldots & 0 \\
0 & 0 & 0 \ldots & 1
\end{array}\right],
$$

$$
\gamma=\left[\begin{array}{c}
0.01 \\
0.01 \\
0.01 \\
\cdot \\
\cdot \\
. \cdot 01
\end{array}\right]
$$

Escreveremos a equação 16, para o vínculo de Igualdade Absoluta na iteração $k+1$ como:

$$
\begin{gathered}
\hat{\mathbf{P}}_{k+1}^{(A I)}=\hat{\mathbf{P}}_{k}-\left[J_{k}^{T} J_{k}+\mu_{A I} A^{T} A+\lambda I\right]^{-1} \times \\
{\left[J_{k}^{T}\left[y^{o}-f\left(\hat{\mathbf{P}}_{k}\right)\right]-\mu_{A I} A^{T}\left(A \mathbf{P}_{k}-\gamma\right)\right] .}
\end{gathered}
$$

\section{Regularizador de Suavidade Global}

$\mathrm{Na}$ Suavidade Global, a diferença entre os valores das estimativas de parâmetros adjacentes espacialmente devem ser muito próximos. Essa informação é incorporada como uma diferença finita entre parâmetros equiespaçados, aproximando as derivadas de primeira ordem através de uma matriz $S$. Fazendose $S=W^{\frac{1}{2}}$, a equação 13 torna-se:

$$
\left\{\begin{array}{c}
\min \phi^{G S}(\mathbf{P})=\|S \mathbf{P}\|^{2}=S^{T} p^{T} S \mathbf{P}, \\
\text { sujeito a } \phi^{o}(\mathbf{P})=\frac{1}{N}\left\|y^{0}-f(\mathbf{P})\right\|^{2}=\delta .
\end{array}\right.
$$

Para o vínculo de Suavidade global, a equação 16 pode, então, ser escrita para a $k$ - ésima+1 iteração como:

$$
\begin{aligned}
\hat{\mathbf{P}}_{k+1}^{G S}= & \hat{\mathbf{P}}_{k}-\left[J_{k}^{T} J_{k}+\mu_{G S} S^{T} S+\lambda I\right]^{-1} \times \\
& {\left[J_{k}^{T}\left[y^{o}-f\left(\hat{\mathbf{P}}_{k}\right)\right]+\mu_{G S} S^{T} S \mathbf{P}_{k}\right] . }
\end{aligned}
$$

Para modelos interpretativos 2D, o operador discreto de primeira derivada precisa ser aplicado tanto na direção horizontal quanto na vertical. Para o modelo da Tabela 1 de nove parâmetros, o operador aplicado na direção horizontal atua nos parâmetros $\mathbf{P}_{1}-\mathbf{P}_{2}, \mathbf{P}_{2}-\mathbf{P}_{3}, \mathbf{P}_{4}-\mathbf{P}_{5}, \mathbf{P}_{5}-\mathbf{P}_{6}, \mathbf{P}_{7}-\mathbf{P}_{8}$ e $\mathbf{P}_{8}-p_{9}$ de modo que a minimização de $\|S \mathbf{P}\|_{2}$ da equação 21 de modo $\mathbf{P}_{1} \approx \mathbf{P}_{2}$, $\mathbf{P}_{2} \approx \mathbf{P}_{3}, \mathbf{P}_{3} \approx \mathbf{P}_{4}, \mathbf{P}_{4} \approx \mathbf{P}_{5}, \mathbf{P}_{5} \approx \mathbf{P}_{6}, \mathbf{P}_{7} \approx \mathbf{P}_{8}, \mathbf{P}_{8} \approx \mathbf{P}_{9}$, ou seja 6 relações de proximidade na direção horizontal entre os 9 parâmetros, que aparecem reunidas na submatriz $(6 \times 9)$ acima da linha divisória da matriz $S$ de 23. O mesmo operador aplicado na direção vertical, por sua vez, atua analogamente nos parâmetros $\mathbf{P}_{1}-\mathbf{P}_{4}, \mathbf{P}_{4}-\mathbf{P}_{7}, \mathbf{P}_{2}-\mathbf{P}_{5}, \mathbf{P}_{5}-\mathbf{P}_{8}, \mathbf{P}_{3}-\mathbf{P}_{6}$ e $\mathbf{P}_{6}-\mathbf{P}_{9}$ permitindo escrever 6 relações de proximidade na direção vertical entre os 9 parâmetros, reunidas na submatriz (6×9) abaixo da linha divisória da matriz $S$ de 23:

Tabela 1: Modelo 2D com 9 parâmetros

\begin{tabular}{c|c|c}
\hline$\downarrow \mathbf{P}_{1} \rightarrow$ & $\downarrow \mathbf{P}_{2} \rightarrow$ & $\downarrow \mathbf{P}_{3}$ \\
\hline$\downarrow \mathbf{P}_{4} \rightarrow$ & $\downarrow \mathbf{P}_{5} \rightarrow$ & $\downarrow \mathbf{P}_{6}$ \\
\hline $\mathbf{P}_{7} \rightarrow$ & $\mathbf{P}_{8} \rightarrow$ & $\mathbf{P}_{9}$
\end{tabular}

Fonte: do autor

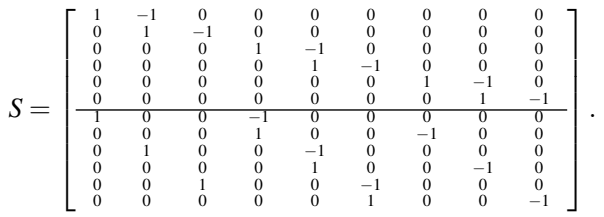

\section{Regularizador de Variação Total}

De forma similar dos anteriores para o regularizador de Variação Total $\phi^{T V}$ :

$$
\left\{\begin{array}{ccc}
\min \phi^{T V}(\mathbf{P}) & = & \|S \mathbf{P}\|^{1}, \\
\text { sujeito a } \phi^{o}(\mathbf{P}) & = & \frac{1}{N}\left\|y^{0}-f(\mathbf{P})\right\|^{2}=\delta .
\end{array}\right.
$$

Pela definição da norma, e do produto $S \mathbf{P}$, é mostrado com mais detalhes em Martins (2009). 
O funcional $\phi^{T V}$ de Variação Total (VT) de (24) é rescrita como :

$$
\phi^{T V}=\sum_{l=1}^{L}\left|\mathbf{P}_{i}-\mathbf{P}_{j}\right|
$$

onde $\mathbf{P}_{i}$ e $\mathbf{P}_{j}$ são o l-ésimo par de parâmetros espacialmente adjacentes e $L$ é o número de total de pares de parâmetros adjacentes ao longo das direções $r$ e z. Entretanto, essa função não é diferenciável quando a diferença entre os parâmetros é igual a zero. Para evitar esse comportamento, a função foi modificada por Acar and Vogel (1994), aproximando as diferenças por:

$$
\left|\mathbf{P}_{i}-\mathbf{P}_{j}\right| \cong\left[\left(\mathbf{P}_{i}-\mathbf{P}_{j}\right)^{2}+\beta\right]^{\frac{1}{2}},
$$

em que $\beta$ é um escalar pequeno e positivo. Para o vínculo de Variação total, a equação 16 pode, então, ser escrita para a $k$ ésima+1 iteração como Martins (2009) :

$$
\begin{array}{r}
\hat{\mathbf{P}}_{k+1}^{T V}=\hat{\mathbf{P}}_{k}-\left[J_{k}^{T} J_{k}+\mu_{V T} S^{T} Q S+\lambda I\right]^{-1} \times \\
{\left[J_{k}^{T}\left[y^{o}-f\left(\hat{\mathbf{P}}_{k}\right)\right]-\mu_{T V} S^{T} \mathbf{q}\right],}
\end{array}
$$

onde a matriz $S$ é expressa em 23 e q é o vetor com a dimensão $(l)$, igual a quantidade de pares de parâmetros que se quer vincular, avaliado em $\mathbf{P}=\mathbf{P}_{k}$, cujo $l$-ésimo elemento é dado por:

$$
\left.\mathbf{q} \equiv \mathbf{q}_{l} \equiv \frac{\mathbf{P}_{i}-\mathbf{P}_{j}}{\left[\left(\mathbf{P}_{i}-\mathbf{P}_{j}\right)^{2}+\beta\right]^{\frac{1}{2}}}\right|_{\mathbf{P}=\mathbf{P}_{k}},
$$

e $Q$ é a matriz diagonal, avaliada em $\mathbf{P}=\hat{\mathbf{P}}(k)$, cujo $l$-ésimo elemento da diagonal é dado por:

$$
\left.Q \equiv Q_{l l} \equiv \frac{\beta}{\left[\left(\mathbf{P}_{i}-\mathbf{P}_{j}\right)^{2}+\beta\right]^{\frac{3}{2}}}\right|_{\mathbf{P}=\mathbf{P}_{k}},
$$

O método não irá penalizar variações bruscas porque, na norma $L 1$, a medida de desajuste entre os pares de parâmetros adjacentes, dará o mesmo valor se a variação dos parâmetros for suave ou se a variação for brusca, o que não é o caso se o mesmo desajuste é medido na norma $L 2$, pois em uma distribuição suave a medida do desajuste é menor, sendo assim favorecida pela minimização desta norma. Mais detalhes sobre essa discussão em Lima et al. (2011).

\section{RESULTADOS}

A contaminação, por ruído, dos dados sintéticos, é utilizador para simular de forma aproxima as situações reais. Desse modo, os dados foram contaminados com ruído de distribuição uniforme com pseudo número aleatórios em um vetor onde o máximo valor é 1 e o mínimo é -1 atribuído em $5 \%$ do valor da observação. $O$ arranjo transmissor-receptor permite gerar os dados constituídos pelos valores da componente vertical do campo magnético no receptor par cada posição da fonte e do receptor. O arranjo fonte-receptor possui $200 \mathrm{~m}$ de extensão em profundidade e $140 \mathrm{~m}$ e $100 \mathrm{~m}$ de distância entre os poços. Foram consideradas 30 e 21 posições para os transmissores e similarmente para os receptores, com separação constante de 10 metros. Isto resulta em 441 e 900 combinaccões diferentes do sistema transmissor-receptor totalizando $2 \times 30 \times 30=1800$ e $2 \times 21 \times 21=882$ observações, pois consideramos a parte real e imaginária do campo, por isso que se multiplica por 2. Estas medidas são compostas pelos campos primário produzido por um dipolo em um meio ilimitado e secundário espalhados pelas heterogeneidades presentes no meio encaixante. Para o campo foi usado a frequência de $100 \mathrm{kHz}$ e heterogeneidades, cujas as condutividades possui valores entre $0.001 \mathrm{~S} / \mathrm{m}$ a $1 \mathrm{~S} / \mathrm{m}$, mantendo o meio encaixante fixo com condutividade de $0.01 \mathrm{~S} / \mathrm{m}$

$\mathrm{Na}$ figura 5 representa o Modelo $\mathrm{B}$. Na Figura 6 tem-se a inversão deste modelo, que possui $14 \times 20$ células totalizando 280 parâmetros e 1800 observações, onde foram utilizado os regularizadores de Suavidade Global e Variação Total. Já na
Figura 2: Modelo de 4 corpos de $20 \mathrm{mX} 20 \mathrm{~m}$ com condutividades $0.02 \mathrm{~S} / \mathrm{m}$ em um meio encaixante de $0.01 \mathrm{~S} / \mathrm{m}$.

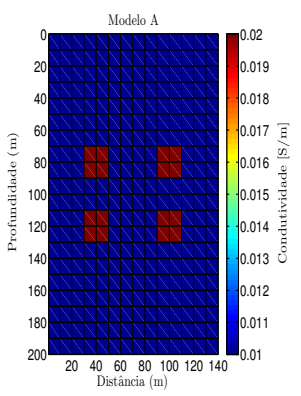

Figura 3: Inversão do Modelo A onde foram utilizados os regularizados: (a) GS com $\mu_{G S}=10^{-22}$ e (b) TV com $\mu_{T V}=10^{-22}$ e $\beta=10^{-5}$.

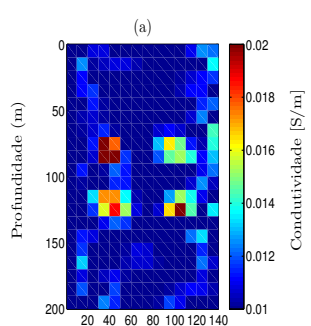

Distância (m)

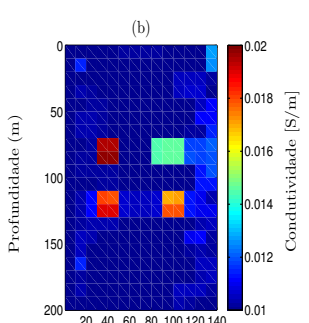

Distância (m)
Figura 4: Inversão do Modelo A onde foram utilizados os regularizadores: (a) GS aliado com 0 regularizador Al com $\mu_{G S}=10^{-22}$ e (b) TV aliado com Al onde $\mu_{T V}=10^{-22}, \beta=10^{-3}$ e $\mu_{A I}=10$.
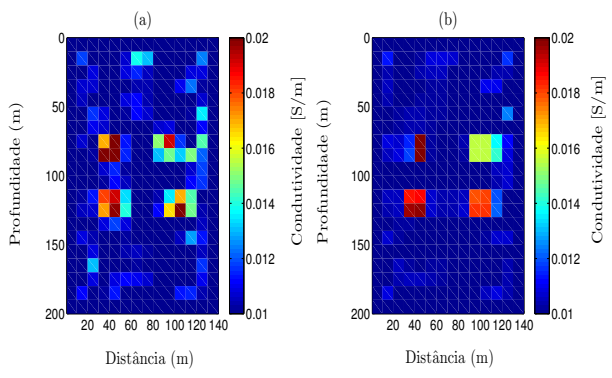

Figura 7 representa a inversão do mesmo modelo com os vínculos de Suavidade Global e Variação Total juntamente com o regularizador de Igualdade Absoluta. Observa-se que a inversão, onde foi utilizado o regularizador de Variação Total, obteve uma melhor estimativa dos corpos condutivos com relação ao vinculo de Suavidade Global. Notou-se também que houve uma melhor estimativa das anomalias utilizando o regularizador de lgualdade Absoluta juntamente aos regularizadores citados anteriormente.

$\mathrm{Na}$ Figura 8 representa o Modelo $\mathrm{C}$. Na Figura 9 representa a inversão deste modelo, que possui $10 \times 20$ células totalizando 200 parâmetros e 882 observações,em que foram utilizados os regularizadores de Suavidade Global e Variação Total. Já na Figura 10 representa a inversão do mesmo modelo com 
Figura 5: Modelo de um corpo com dimensão $60 \mathrm{mx} 10 \mathrm{~m}$ com condutividade $1 \mathrm{~S} / \mathrm{m}$ em um meio encaixante de $0.01 \mathrm{~S} / \mathrm{m}$.

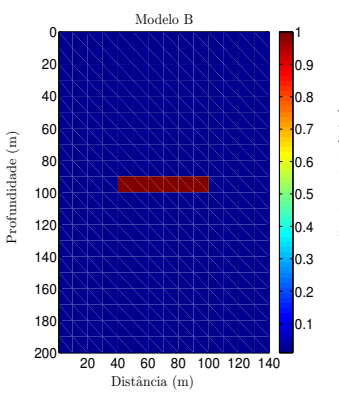

Figura 6: Inversão do Modelo $\mathrm{B}$ em que foram utilizados os regularizadores: (a) GS aliado com o vínculo $\mathrm{Al}$, em que $\mu_{G S}=$ $10^{-26}$ e $\mu_{A I}=10$ e (b) TV aliado com o vínculo $\mathrm{Al}$, em que $\mu_{T V}=10^{-23}, \beta=10^{-2}$ e $\mu_{A I}=10$.
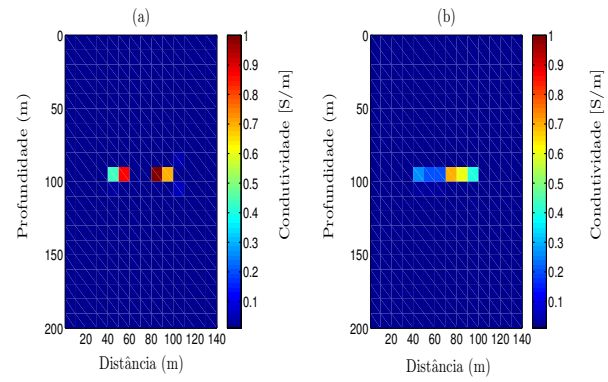

Figura 7: Inversão do Modelo B em que foram utilizados os regularizadores : (a) GS aliado com o vínculo Al, em que $\mu_{G S}=$ $10^{-24}$ e $\mu_{A I}=10$ e (b) TV aliado com o regularizador IA, em que $\mu_{T V}=10^{-23}, \beta=10^{-2}$ e $\mu_{I A}=10$.
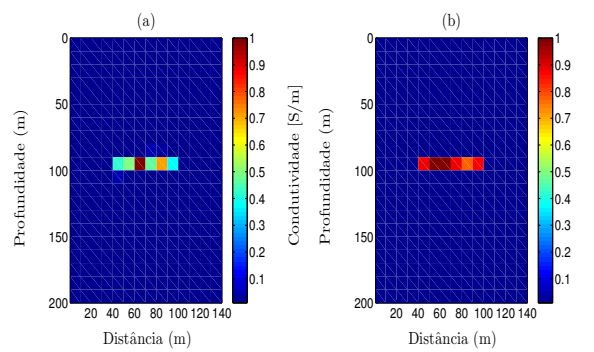

os Vínculos de Suavidade Global e Variação Total juntamente com o regularizador de Igualdade Absoluta. Observa-se que a inversão utilizando o regularizador de Variação Total obteve melhor estimativa dos corpos condutivos com relação ao vinculo de Suavidade Global. Notou-se também que houve uma melhor recuperacão dos corpos condutivos utilizando o regularizador de Igualdade Absoluta auxiliando os regularizadores citados anteriormente.

Na figura 11 representa o Modelo D . Na Figura 12 representa a inversão do desse modelo, que possui $10 \times 20$ células totalizando 200 parâmetros e 882 observações, em que foram utilizados os regularizadores de Suavidade Global e Variação Total. Já na Figura 13 representa a inversão do mesmo modelo com os
Figura 8: Modelo de um corpo com dimensão $20 \mathrm{mx} 80 \mathrm{~m}$ e condutividade $0.1 \mathrm{~S} / \mathrm{m}$ em um meio encaixante de 0.01 $\mathrm{S} / \mathrm{m}$.

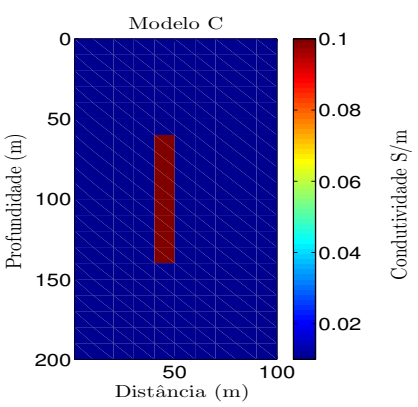

Figura 9: Inversão do Modelo $C$ em que foram utilizados os regularizadores: (a) GS com $\mu_{G S}=10^{-19}$ e (b) TV com $\mu_{T V}=$ $10^{-18}$ e $\beta=10^{-2}$
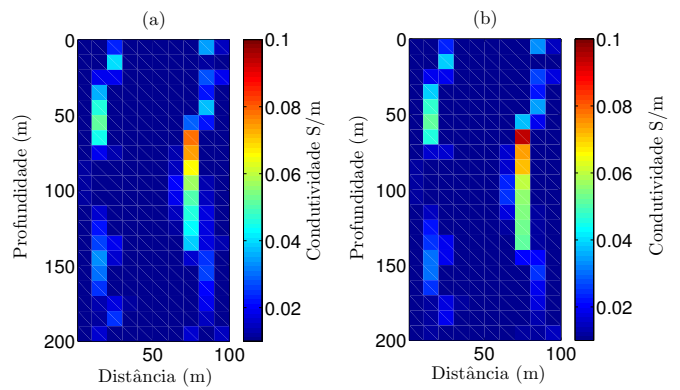

Figura 10: Inversão do Modelo $C$ em que foram utilizados os regularizadores: (a) GS com Al onde $\mu_{G I}=10^{-19}$ e (b) TV com Al onde $\mu_{T V}=10^{-18}, \beta=10^{-2}$ e $\mu_{A I}=10$.
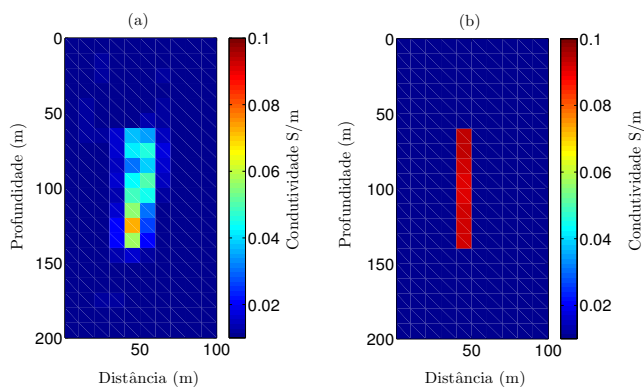

Fonte: Do autor

Vínculos de Suavidade Global e Variação Total juntamente com o Regularizador de Igualdade Absoluta. Observa-se que a inversão utilizando o regularizador de Variação Total obteve melhor estimativa dos corpos condutivos, como sua condutividade e localização, com relação ao vinculo de Suavidade Global. Percebe-se também que houve uma melhor estimativa dos alvos condutivos utilizando o regularizador Igualdade absoluta auxiliando os regularizadores citados anteriormente. 
Figura 11: Modelo de um corpo inclina com condutividade 0.1 $\mathrm{S} / \mathrm{m}$ e um corpo retangular com condutividade $0.001 \mathrm{~S} / \mathrm{m}$ em um meio encaixante de $0.01 \mathrm{~S} / \mathrm{m}$.

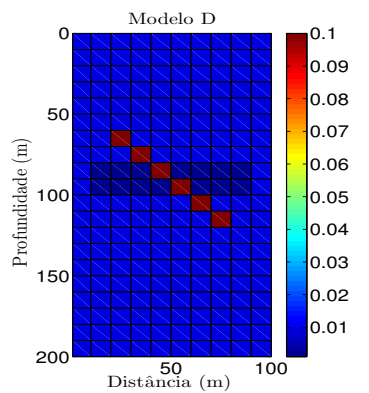

Fonte: Do autor

Figura 12: Inversão do Modelo $D$ em que foram utilizados os regularizadores: (a) GS com $\mu_{G S}=10^{-20}$ e (b) TV com $\mu_{T V}=$
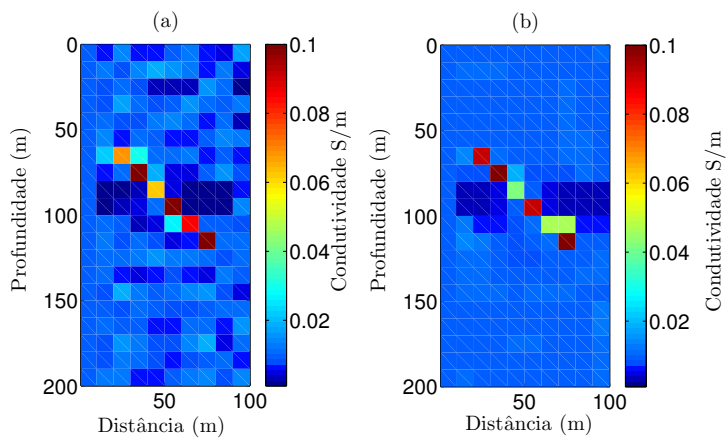

Figura 13: Inversão do Modelo $D$ em que foram utilizados os regularizadores (a) GS com Al $\mu_{S G}=10^{-21}$ e (b) TV com Al $\mu_{T V}=$ $10^{-19}, \beta=10^{-2}$ e $\mu_{A I}=10$.
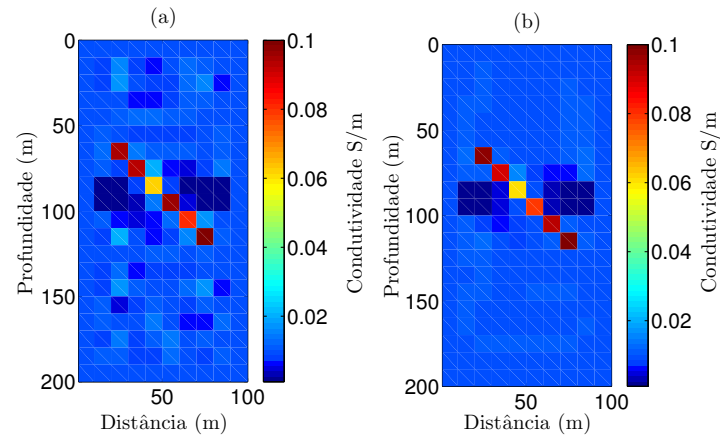

\section{Conclusões}

Este trabalho desenvolveu uma metodologia do problema direto e inverso dos dados de tomografia eletromagnética poco-a-poço. No problema direto usamos o método de elementos finitos para a solução numérica da equação de Helmholtz. Os métodos apresentados neste trabalho tiveram seu desempenho avaliado através de dados sintéticos atribuídos com ruído pseudo aleatório de distribuição uniforme com $5 \%$ da observação, de modo que, os dados tenham maior proximidade a situações reais. Já no problema inverso discutimos o uso dos funcionais estabilizadores de Suavidade Global , de Variação Total e de Igualdade Absoluta.
O primeiro funcional usa uma suavização na norma $L_{2}$, enquanto - segundo usa uma suavização na norma $L_{1}$, que aceita variações abruptas entre os parâmetros adjacentes. Nossos resultados mostram que o uso do estabilizador de Variação Total obteve boa estimativa da geometria e da condutividade dos corpos, tanto para pequenos, quanto para grandes contrastes de condutividades entre os alvos e o meio encaixante. Observamos também que o regularizador de Variação Total apresentou uma melhor estimativa dos parâmetros, comparado ao uso do regularizador de Suavidade Global. Em boa parte dos modelos sintéticos utilizados neste trabalho, obtivemos uma melhor estimativa dos corpos, quando usamos regularizadores de Suavidade Global e de Variação Total com o auxílio do vínculo de lgualdade Absoluta nas bordas da malha de inversão.

\section{Acknowledgments}

Agradeço ao CPGF pelo espaço cedido para o desenvolvimento desse trabalho e o CNPQ pelo apoio financeiro.

\section{Referências}

Acar, R., \& Vogel, C. R., 1994, Analysis of bounded variation penalty methods for ill-posed problems: Inverse Problems, 10 , no. $6,1217$.

Alumbaugh, D. L., \& Morrison, H. F., 1995, Theoretical and practical considerations for crosswell electromagnetic tomography assuming a cylindrical geometry: Geophysics, 60 , no. 3, 846-870.

Bard, Y., 1974, Nonlinear parameter estimation: Academic Press.

French, W., Marcoux, M., \& Matzuk, T., 1973, Technical limitation of seismic holography: Geophysics, 38, 16-22.

Lima, W. A., Martins, C. M., Silva, J. B. C., \& Barbosa, V. C. F., 2011, Total variation regularization for depth-to-basement estimate: Part 2 - physicogeologic meaning and comparisons with previous inversion methods: Geophysics, 76, no. 1, I13120.

Martins, C. M., 2009, Inversão gravimétrica do relevo 3d de bacias sedimentares e da variação da densidade usando informação a priori sobre o ambiente geológico.: Ph.D. thesis, Observatório Nacional.

Schneider, W. A., \& French, W. S., 1976, Computer migration of oblique seismic reflection profiles: Geophysics, 41, no. 4, 776777.

shan ; Xu Shao-hui, W. R., 1979, digital holography applied to borehole electromagnetic wave exploration:.

Silva, J. B. C., Medeiros, W. E., \& Barbosa, V. C. F., 2001, Pitfalls in nonlinear inversion: pure and applied geophysics, 158, no. 5-6, 945-964.

Souza, V. C. T., 2001, Tomografia eletromagnética usando vínculos absolutos: Master's thesis, Universidade Federal do Pará.

Wade, R. K. M. M. K. G., 1979, Reconstructive tomography and applications to ultrasonics:, 67, 567-587.

Ward, S. H., \& Hohmann, G. W., 1988, Electromagnetic theory for geophysical applications, volume 1 of Investigations in Geophysics Society of Expolration Geophysicists SEG.

Wilt, M. J., Alumbaugh, D. L., Morrison, H. F., Becker, A., Lee, K. H., \& Deszcz-Pan, M., 1995, Crosswell electromagnetic tomography; system design considerations and field results: Geophysics, 60, no. 3, 871-885. 